6. Kim J, Mierzwinski-Urban M. Ketamine for treatment-resistant depression or post-traumatic stress disorder in various settings: a review of clinical effectiveness, safety, and guidelines. CADTH rapid response reports. March 1, 2017. Available at: https://www.cadth.ca/sites/default/files/pdf/htis/2017/ RC0855\%20Ketamine\%20for\%20Resistant\%20Depression\%20Final.pdf. Accessed December 11, 2019.

7. Papadimitropoulou K, Vossen C, Karabis A, Donatti C, Kubitz N. Comparative efficacy and tolerability of pharmacological and somatic interventions in adult patients with treatment-resistant depression: a systematic review and network meta-analysis. Curr Med Res Opin. 2017;33(4):701-11.

8. Wilkinson ST, Toprak M, Turner MS, Levine SP, Katz RB, Sanacora G. A survey of the clinical, off-label use of ketamine as a treatment for psychiatric disorders. Am J Psychiatry. 2017;174(7):695-96.

9. Popova V, Daly EJ, Trivedi M, et al. Efficacy and safety of flexibly dosed esketamine nasal spray combined with a newly initiated oral antidepressant in treatment-resistant depression: a randomized double-blind active-controlled study. Am J Psychiatry. 2019;176(6):428-38.

10. Fedgchin M, Trivedi M, Daly E, et al. Randomized, double-blind study of fixed-dosed intranasal esketamine plus oral antidepressant vs. active control in treatment-resistant depression. Poster presented at: 9th Biennial Conference of the International Society for Affective Disorders and the Houston Mood Disorders Conference; September 20-22, 2018; Houston, TX. Available at: https://www.psychcongress.com/posters/randomized-doubleblind-study-fixed-dose-esketamine-nasal-spray-plus-oral-antidepressant-vs. Accessed December 9, 2019.

11. Ochs-Ross R, Daly E, Zhang Y, et al. Efficacy and safety of esketamine nasal spray plus an oral antidepressant in elderly patients with treatmentresistant depression. Am J Geriatr Psychiatry. 2019;27(3S):S139-40.

12. Daly EJ, Trivedi MH, Janik A, et al. Efficacy of esketamine nasal spray plus oral antidepressant treatment for relapse prevention in patients with treatment-resistant depression: a randomized clinical trial. JAMA Psychiatry. June 5, 2019 [Epub ahead of print].

13. Rush AJ, Trivedi MH, Wisniewski SR, et al. Acute and longer-term outcomes in depressed outpatients requiring one or several treatment steps: a STAR*D report. Am J Psychiatry. 2006;163(11):1905-17.

14. Institute for Clinical and Economic Review. ICER value assessment framework. Available at: https://icer-review.org/methodology/icers-methods/ icer-value-assessment-framework-2/. Accessed December 9, 2019.

\title{
Dissociating the Clinical Role and Economic Value of Intranasal Esketamine David Dadiomov, PharmD, BCPP
}

\section{COMMENTARY}

the first study to evaluate the use of ketamine for depression was published and disrupted how we think about depression treatment. ${ }^{1}$ The study was conducted with intravenous ketamine and has been replicated many times in robust study settings. ${ }^{2}$ Although ketamine had been used as an anesthetic since the 1960s, it was not until the 2000s that the psychiatric utility of ketamine gained mainstream attention. ${ }^{3}$ The majority of the evidence base for the antidepressive and antisuicidal effects of ketamine were documented with intravenous or intramuscular racemic ketamine. ${ }^{2,4}$ Esketamine, an S-enantiomer, was given breakthrough therapy designation by the U.S. Food and Drug Administration (FDA) in 2013 for the treatment of treatment-resistant depression (TRD) and was approved as an intranasal formulation for this indication in 2019.

In June 2019, the Institute for Clinical and Economic Review (ICER) published its final evidence report on the clinical and economic implications of intranasal esketamine approval. ${ }^{5}$

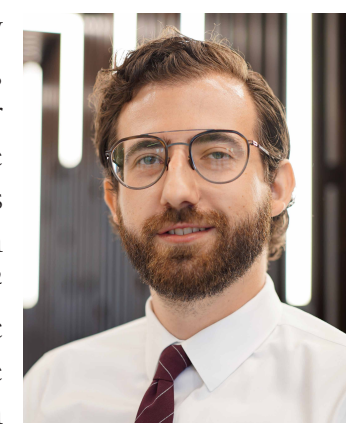

The final report notes that intranasal esketamine provides a clinical benefit to patients that have TRD and are being treated with a background antidepressant as compared with a background antidepressant alone. Of course, there are no direct comparison trials available with other modalities for treating TRD (i.e., antidepressant augmentation, transcranial magnetic stimulation, electroconvulsive therapy, or parenteral racemic ketamine). Although the clinical utility of this new therapy is positive, the ICER report demonstrates that the value and pricing of the new intranasal esketamine product may be less than ideal.

The ICER report uses a standard metric of cost-effectiveness, the quality-adjusted life-year (QALY). Typically, medications with a cost per QALY of \$50,000-150,000 are considered a reasonable value. ${ }^{6}$ The new intranasal esketamine product delivers a cost per QALY of $\$ 198,000$. The ICER analysis used a de novo decision analytic model with clinical inputs derived from the TRANSFORM-1 and -2 and SUSTAIN-1 and -2 trials of intranasal esketamine and from the STAR*D trial (the largest trial of real-world antidepressant therapy, augmentation, and switching). There are certainly some important factors that can be attributed to the high cost per QALY that was reported; 
however, a critical assessment of the pricing of the intranasal esketamine product is warranted.

The discovery that ketamine can have profound effects on the treatment of depression and suicidality in patients with TRD is truly a breakthrough in the treatment of mental health disorders. The predominant modality for treating depression has remained mostly unchanged since antidepressant therapy first emerged with monoamine oxidase inhibitors (MAOIs) in the 1950s. Through MAOIs, tricyclic antidepressants, selective serotonin reuptake inhibitors, serotonin-norepinephrine reuptake Inhibitors, a few atypical agents, and several "me-too" drugs, the mode of action revolved around the modulation of the monoamines (serotonin, norepinephrine, or dopamine). Although effective augmentation strategies such as lithium and triiodothyronine may work outside of the monoamine hypothesis of depression, new modalities are clearly needed, given the significant proportion of patients that do not achieve response or remission with current therapies. ${ }^{7}$

Before the FDA approval of the esketamine product, treatment of patients with TRD with ketamine was in a contentious state. There is considerable clinical efficacy data on the depressive symptoms of TRD, as well as on acute suicidality. Before this, the pharmacotherapies with evidence of antisuicidal properties included lithium and clozapine, which have their own set of limitations. ${ }^{8}$

In addition, the cumulative clinical experience with ketamine was growing tremendously-arguably disproportionately from the published short-term trials. Many private practices opened and began offering ketamine treatments (in a variety of formulations) off-label for treatment of (primarily) depression. This was problematic for a variety of reasons. Many of these "new" providers of ketamine were not formally trained to treat psychiatric disorders (many were trained in anesthesia or emergency medicine). ${ }^{9}$ Some practices may have used different formulations or doses of ketamine than had been previously reported to be efficacious. Throughout these years, there were no long-term efficacy or safety outcomes published, despite some providers using ketamine as a chronic depression treatment. Infusions of ketamine could be repeated monthly or bimonthly, and patients would pay out of pocket (ranging from $\$ 500$ to $\$ 1,000$ per treatment).

Ketamine's mechanism of action is not entirely understood. Ketamine has primarily been considered an N-methyl-Daspartate receptor (NMDA) antagonist, and many initially attributed the antidepressant activity to this pharmacologic property. More recent evidence offers a few other possibilities for the antidepressant action. While NMDA receptors may play a role in the clinical effects, it is important to note that other NMDA receptor antagonists have not been effective for treating depressionacutely. The antidepressanteffects of ketamine maybea result of a number of different factors, such as a-amino-3-hydroxy-5methyl-4-isoxazolepropionic acid (AMPA) receptor modulation, changes in neuroplasticity, mystical experience, brain-derived neurotrophic factor, and mammalian target of rapamycin (mTOR). To complicate matters further, there is evidence that different enantiomers of ketamine may have differing roles in the management of depression, and it is likely that metabolites of ketamine, such as norketamine and hydroxynorketamine, may play a critical role. ${ }^{10}$

Although the clinical trials for intranasal esketamine demonstrated an improvement in Montgomery-Åsberg Depression Rating Scale (MADRS) scores over a placebo nasal spray, it should be noted that there was a high response rate for placebo in these studies. This may have been one of the factors leading to a smaller than expected mean difference in MADRS scores. The least squares mean difference in MADRS scores was -4.0 in the TRANSFORM trial in favor of esketamine over placebo. Other considerations include dosing differences in an intranasal product, as well as potency of the S-enantiomer as compared with racemic ketamine, and implications of metabolite formation. ${ }^{11}$ These factors lead to a reduced (although still clinically significant) difference in treatment from placebo but likely contribute to the high cost per QALY that was reported as well.

On value, we still find ourselves in a contentious state. An important advancement in depression treatment was made when the FDA approved intranasal esketamine. However, an objective analysis of the cost has deemed this therapy to be more expensive than a previously determined threshold for cost-effectiveness. While there are certainly improvements that can be made to the model that analyzed the cost-effectiveness of esketamine and considerations as to the clinical performance of this medication, the pricing of the esketamine product is concerning for patients and providers of mental health.

Given the current state, it appears that the onus will now fall on payers to determine how accessible intranasal esketamine will be. The final ICER evidence report lists some suggestions for management. At least one of these suggestions is counterproductive. The report suggests to restrict esketamine therapy from patients with active substance use and has wording that patients with 1 year of abstinence from substances may particularly benefit. ${ }^{5}$ To the contrary, there is evidence to support that ketamine may actually be beneficial in the treatment of various substance use disorders. ${ }^{12,13}$ Payers, administrators, and clinicians should be cautious in making the assumption 
that because a substance has been used recreationally that a prescription version is prone to misuse, as has been observed with opioids or stimulants.

A productive suggestion given in the ICER report was to consider the coverage of racemic ketamine formulations (perhaps with provider and patient criteria) as an alternative to esketamine treatment. ${ }^{5}$ Although no comparative trials have been conducted, there is a large and increasing amount of data on parenteral racemic ketamine for suicidality and depression. ${ }^{14}$ As mentioned previously, there is evidence that both isomers of ketamine have therapeutic properties thus adding value to managed care companies. The cost of racemic ketamine is very low; even considering the administration and monitoring costs, it becomes a much better value for patients and increases the accessibility of this therapy to a broader population.

The designation of intranasal esketamine as a breakthrough therapy is rightfully earned as a novel mechanism to treat depression and as a pharmacotherapy that can acutely treat symptoms. The enthusiasm surrounding this treatment should not detract from the remaining questions to be addressed. The factors that lead to successful treatment, such as active metabolites, enantiomer-specific effects, routes of administration, and downstream neural mechanisms of action, should continue to be studied. These answers will further help determine the value of these treatments in the current armamentarium of depression treatments. Although esketamine is not currently approved for acute suicidality, there is evidence that intranasal esketamine and racemic ketamine may be promising treatments for this condition. ${ }^{4,15}$ Assessing this data in conjunction with overall depression treatment may enhance the value for these treatments.

Depression is one of the leading causes of disability in the United States. ${ }^{16}$ While great strides are being made to treat this condition, payers, clinicians, and pharmaceutical companies should ensure that these treatments are accessible to the patients that need it by demonstrating value and sustainability.

\section{Authors}

DAVID DADIOMOV, PharmD, BCPP, University of Southern California School of Pharmacy, Los Angeles.

AUTHOR CORRESPONDENCE: David Dadiomov, PharmD, BCPP, Assistant Professor of Clinical Pharmacy, University of Southern California School of Pharmacy, 1985 Zonal Ave., Los Angeles, CA 90027.E-mail: dadiomov@usc.edu.

\section{DISCLOSURES}

No funding supported the writing of this commentary. The author has nothing to disclose.

\section{REFERENCES}

1. Berman RM, Cappiello A, Anand A, et al. Antidepressant effects of ketamine in depressed patients. Biol Psychiatry. 2000;47(4):351-54.

2. Serafini G, Howland RH, Rovedi F, Girardi P, Amore M. The role of ketamine in treatment-resistant depression: a systematic review. Curr Neuropharmacol. 2014;12(5):444-61.

3. Grady SE, Marsh TA, Tenhouse A, Klein K. Ketamine for the treatment of major depressive disorder and bipolar depression: a review of the literature. Ment Health Clin. 2017;7(1):16-23.

4. Dadiomov D, Lee K. The effects of ketamine on suicidality across various formulations and study settings. Ment Health Clin. 2019;9(1):48-60,

5. Institute for Clinical and Economic Review. Esketamine for the treatment of treatment-resistant depression: effectiveness and value. Final evidence report. June 20, 2019. Available at: https://icer-review.org/wp-content/ uploads/2018/10/ICER_TRD_Final_Evidence_Report_062019.pdf. Accessed December 10, 2019.

6. Neumann PJ, Cohen JT, Weinstein MC. Updating cost-effectivenessthe curious resilience of the \$50,000-per-QALY threshold. N Engl J Med. 2014;371(9):796-97.

7. Gaynes BN, Warden D, Trivedi MH, Wisniewski SR, Fava M, Rush AJ. What did STAR*D teach us? Results from a large-scale, practical, clinical trial for patients with depression. Psychiatr Serv. 2009;60(11):1439-45.

8. Griffiths JJ, Zarate CA Jr, Rasimas JJ. Existing and novel biological therapeutics in suicide prevention. Am J Prev Med. 2014:47(3 Suppl 2):S195-203.

9. Wilkinson ST, Toprak M, Turner MS, Levine SP, Katz RB, Sanacora G. A survey of the clinical, off-label use of ketamine as a treatment for psychiatric disorders. Am J Psychiatry. 2017;174(7):695-96.

10. Zanos P, Gould TD. Mechanisms of ketamine action as an antidepressant. Mol Psychiatry. 2018;23(4):801-11.

11. Zhang K, Hashimoto K. An update on ketamine and its two enantiomers as rapid-acting antidepressants. Expert Rev Neurother. 2019;19(1):83-92.

12. Dakwar E, Levin F, Hart CL, et al. A single ketamine infusion combined with motivational enhancement therapy for alcohol use disorder: a randomized midazolam-controlled pilot trial. Am J Psychiatry. December 2, 2019 [Epub ahead of print]

13. Dakwar E, Nunes EV, Hart CL, et al. A single ketamine infusion combined with mindfulness-based behavioral modification to treat cocaine dependence: a randomized clinical trial. Am J Psychiatry. 2019;176(11):923-30.

14. Wilkinson ST, Ballard ED, Bloch MH, et al. The effect of a single dose of intravenous ketamine on suicidal ideation: a systematic review and individual participant data meta-analysis. Am J Psychiatry. 2018;175(2):150-58.

15. Canuso CM, Singh JB, Fedgchin M, et al. Efficacy and safety of intranasal esketamine for the rapid reduction of symptoms of depression and suicidality in patients at imminent risk for suicide: results of a double-blind, randomized, placebo-controlled study. Am J Psychiatry. 2018;175(7):620-30.

16. Friedrich MJ. Depression is the leading cause of disability around the world. JAMA. 2017;317(15):1517. 